\title{
Application of Neural Networks to MRAC for the Nonlinear Magnetic Levitation System*
}

\author{
Agus TRISANTO**, Muhammad YASSER ${ }^{* *}$, Ayman HAGGAG*, Jianming LU** \\ and Takashi YAHAGI**
}

\begin{abstract}
This paper investigates the application of neural networks (NNs) to conventional model reference adaptive control (MRAC) for controlling the real plant of the nonlinear magnetic levitation system. In the conventional MRAC scheme, the controller is designed to realize the plant output convergence to the reference model output based on the assumption that the plant can be linearized. This scheme is effective for controlling a linear plant with unknown parameters in the ideal case. However, it may not be assured to succeed in controlling a nonlinear plant with unknown structures in the real case. We incorporate a neural network in the MRAC to overcome this problem. The control input is given by the sum of the output of the adaptive controller and the output of the NN. The NN is used to compensate for the nonlinearity of the plant that is not taken into consideration in the conventional MRAC. We developed an efficient method for calculating the sensitivity of the plant that is utilized in the $\mathrm{NN}$ to perform the backpropagation algorithm very efficiently. The plant of the magnetic levitation system has inherent strong nonlinearities due to the natural properties of the magnetic fields and uncertainties. Therefore, to confirm the effectiveness of our proposed controller, we implemented our proposed controller in real time on an experimental test bed of a magnetic levitation system. Finally, experimental results verified that the proposed control strategy has the advantages of tracking desired output perfectly and reducing the error.
\end{abstract}

Key Words: MRAC, Neural Networks, Magnetic Levitations, Nonlinear Control

\section{Introduction}

Recently, adaptive control has been developed to fulfill demanding industrial applications. The primary reason for using adaptive control in industrial is to overcome the difficulties of creating a controlled system that could work over a wide range of operating conditions because of the effects of process variations and disturbances. One of the important classes of adaptive control is model reference adaptive control (MRAC) that has been proven to be one of the popular methods in the growing field of adaptive control, particularly for practical applications ${ }^{(1)}$.

Usually an MRAC is implemented by a reference model, an adjustment mechanism, a controller and a plant $^{(1)-(4)}$. In the conventional MRAC scheme, the controller is designed to realize a plant output convergence to

* Received 21st October, 2006 (No. 05-4230)

** Graduate School of Science and Technology, Chiba University, 1-33 Yayoi-cho, Inage-ku 263-8522, Japan.

E-mail: atrisanto@graduate.chiba-u.jp reference model output based on the assumption that the plant can be linearized. Therefore, this scheme is effective for controlling a linear plant with unknown parameters in the ideal case, but it may not be assured to succeed in controlling a nonlinear plant with unknown structures in the real case ${ }^{(1),(4),(5)}$.

The Neural Networks (NNs) are able to learn from example, recognize data, identify pattern, and determine approximation of a nonlinear function, and this makes it a prime candidate to be utilized in the area of nonlinear control systems. The NN is required to better control complex uncertainty dynamical systems and to improve performance of the conventional control method ${ }^{(6)-(8)}$. Many researchers have employed the $\mathrm{NN}$ in their control design ${ }^{(5),(7)-(13)}$. For example, the neural networks have been applied in a direct adaptive control for unknown nonlinear systems ${ }^{(9)}$. Also, the possibility of using NN in three control architectures (MRAC, model predictive control and feedback linearization control) have been proven $^{(5),(11)-(13)}$. Using neural networks technologies to 
the MRAC structure was found to provide a greatly improved performance over conventional approaches ${ }^{(5),(12)}$. The NN has been utilized as adaptive controller with fixed gain to control nonlinear plant ${ }^{(12)}$. The effectiveness of using NN in MRAC for nonlinear systems has been verified by simulation studies in Ref. (5). So far, most of the results in neural network researches were based on simulation studies. Implementing the theory of the $\mathrm{NN}$ on the plant at real time is a difficult problem, because there are many aspects that have to be considered such as uncertainties noises, dynamic characteristics of the plants, specifications of the plant systems, sensors and actuators calibrations, lifetime of the components of plants, and environmental conditions. In this research, we apply a neural network strategy in the MRAC to control the experimental magnetic levitation system test bed, and also we develop the method in reference 5 with the theoretical consideration about the stability of the controller.

Magnetic levitation systems have a practical importance in many engineering systems such as frictionless bearings, levitation of high speed trains and vibration isolation tables in semiconductor manufacturing. On the other hand, the magnetic levitation systems have inherent strong nonlinearities due to the natural properties of magnetic fields and uncertainties ${ }^{(14),(19)}$. Therefore, we would like to verify the effectiveness of our proposed control system on the experimental magnetic levitation system in real time. Controlling the magnetic levitation system at real time is a difficult control problem. It is not assured to succeed in estimating parameters of nonlinear plant using only conventional MRAC. Hence, the multilayer backpropagation networks has been utilized in our proposed control method because of its inherent nonlinear mapping capabilities ${ }^{(8)}$. The control input is given by the sum of the output of the adaptive controller and the output of NN. The NN is used to compensate for the nonlinearity of the plant that is not taken into consideration in the conventional MRAC. The role of the MRAC is to perform the model matching for the uncertain linearized system to a given reference model. One of the characteristic features of the proposed structure is to give an efficient method for calculating the derivative of the system output with respect to the input using one identified parameter in the linearized model and the internal variables of the NN, which enables performing the backpropagation algorithm very efficiently. The neural network weights are adjusted by multilayer backpropagation algorithm which is carried out online.

This paper is organized as follows. The concept of the conventional MRAC is presented in section 2. Section 3, describes the proposed control scheme. Stability analysis of the system is discussed in section 4. Section 5, explains the experimental apparatus of the magnetic levitation system. Then in section 6, experimental results are carried out to verify the effectiveness of our proposed control schemes. Finally, conclusions of the design scheme are given in section 7 .

\section{Conventional MRAC}

In this section, we briefly describe a linear discretetime conventional MRAC, where the controller is designed to realize a plant output $y(k)$ that converges to reference model output $y_{m}(k)$. In this method based on the assumption that the characteristics of plant can be linearized, then with a linear control principle a plant output can be controlled. The basic structure of the MRAC is shown in Fig. 1. The output of the reference model is compared to the output of the plant. The error between the reference model output and the plant output is used to estimate parameters of the plant, then based on the parameters estimated the controller is constructed.

\subsection{Parameter estimation}

We can write the relation between the plant output and input of a linear discrete time system as ${ }^{(5),(15)}$ :

$$
\begin{aligned}
y(k) & =-\sum_{i=1}^{n} a_{i} y(k-i)+\sum_{j=0}^{m} b_{j} u(k-d-j) \\
& =\alpha^{T} X(k)
\end{aligned}
$$

where the input and the output of plant are denoted by $u(k)$ and $y(k)$ respectively, $T$ denotes the transpose, $\alpha=$ $\left[a_{1}, a_{2}, \ldots, a_{n}, b_{0}, b_{1}, \ldots, b_{m}\right]^{T}$, and $X(k)=[-y(k-1),-y(k-$ $2), \ldots,-y(k-n), u(k-d), u(k-d-1), \ldots, u(k-d-m)]^{T}, n$, and $m$ are the numbers of past outputs and inputs of the plant depending on the plant order, $d$ represent known time delay. Here, $\alpha$ represents the unknown parameters of the plant and need to be estimated. This can be accomplished by using an identification model described by the equation

$$
\bar{y}(k)=\bar{\alpha}^{T}(k) X(k)
$$

where $\bar{\alpha}(k)=\left[\bar{a}_{1}(k), \bar{a}_{2}(k), \ldots, \bar{a}_{n}(k), \bar{b}_{0}(k), \bar{b}_{1}(k), \ldots, \bar{b}_{m}(k)\right]^{T}$, $\bar{y}(k)$ is the estimated value of $y(k)$ at the time $k$, and $\bar{\alpha}(k)$ represents the unknown parameters that will be estimated.

The parameter adjustment law, which ensures that the estimated parameters can converge to their true values, is given by ${ }^{(5),(15),(16)}$

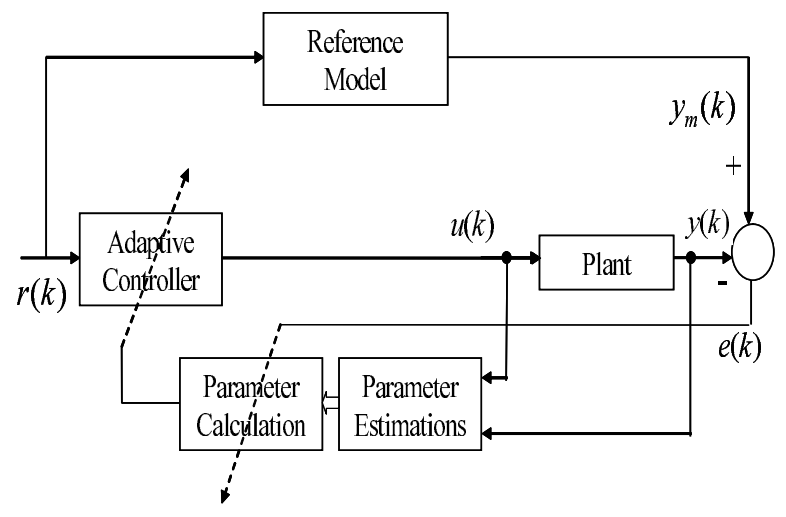

Fig. 1 Conventional MRAC 


$$
\begin{aligned}
\bar{\alpha}(k)= & \bar{\alpha}(k-1) \\
& -\frac{\Gamma(k-1) X(k)\left[\bar{\alpha}^{T}(k-1) X(k)-y(k)\right]}{1+X^{T}(k) \Gamma(k-1) X(k)} \\
\Gamma(k)= & {\left[\Gamma(k-1)-\frac{\Gamma(k-1) X(k) X^{T}(k) \Gamma(k-1)}{1+X^{T}(k) \Gamma(k-1) X(k)}\right] } \\
\Gamma(0)= & I
\end{aligned}
$$

\subsection{Controller}

The control system attempts to make the plant output $y(k)$ match the reference model output $y_{m}(k)$ asymptotically, i.e.

$$
\lim _{k \rightarrow \infty}\left|y_{m}(k)-y(k)\right| \leq \varepsilon
$$

for some specified constant $\varepsilon \geq 0$. Furthermore, (1) can be rewritten as

$$
A(z) y(k)=z^{-d} B(z) u(k)
$$

where $A(z)=1+\sum_{i=1}^{n} a_{i} z^{-i}, B(z)=\sum_{j=0}^{m} b_{j} z^{-j}$, and $z^{-i}$ is the backward shift operator, $z^{-1} y(k)=y(k-1), d$ represents the known time delay. Furthermore, $u(k)$ and $y(k)$ are the plant input and output, respectively. The polynomials $A(z)$ and $B(z)$ are coprime (but have unknown coefficients, and $\left.b_{o} \neq 0\right)$ and the upper bounds of the degrees of $A(z)$ and $B(z)$ are known.

Let $D(z)=1+d_{1} z^{-1}+\ldots+d_{n} z^{-n}$ be an asymptotically stable polynomial, then there exist unique polynomials $R(z)$ and $S(z)$ which satisfy :

$$
D(z)=A(z) S(z)+z^{-d} R(z)
$$

where $A(z)$ is the polynomial in (8), and $S(z)=1+\sum_{i=1}^{d-1} s_{i} z^{-i}$ and $R(z)=\sum_{i=0}^{n-1} r_{i} z^{-i}$

Substituting (8) to (9), and add $D(z) y_{m}(k)$ to the both sides, we obtain

$$
\begin{aligned}
D(z)\left[y_{m}(k)-y(k)\right]= & -z^{-d} B(z) S(z) u(k)-z^{-d} R(z) y(k) \\
& +D(z) y_{m}(k)
\end{aligned}
$$

It is clear that if $\lim _{k \rightarrow \infty}\left|y_{m}(k)-y(k)\right|=0$, then the control objective can be achieved. Consequently, the control input is given by

$$
u(k)=\frac{D(z) y_{m}(k+d)-R(z) y(k)}{B(z) S(z)}
$$

Let $D(z)=1$, then the control input $u(k)$ in the case of unknown parameters is given by

$$
u(k)=\frac{y_{m}(k+d)-\bar{R}(z) y(k)}{\bar{B}(z) \bar{S}(z)}
$$

where $\bar{R}(z), \bar{B}(z)$, and $\bar{S}(z)$ are the estimates of $R(z), B(z)$ and $S(z)$, respectively. Furthermore $\bar{B}(z)$ can be obtained from $\bar{\alpha}$ in (4), then $\bar{R}(z)$ and $\bar{S}(z)$ can be calculated by diophantine equation [see Appendix 1].

The control input $u(k)$ in (12) is effective in controlling a linear plant, with unknown parameters, but is not assured to succeed in controlling a nonlinear plant.

\section{MRAC Using NN}

When the input and output characteristics of the system is nonlinear, it is not possible to express it as in (1). Therefore, we use the following equation to express nonlinear discrete time system

$$
y(k)=G(y(k-1), \ldots, y(k-n), u(k-d), \ldots, u(k-d-m))
$$

where $G($.$) is the unknown nonlinear function, y(k)$ is the plant output, $u(k)$ is the control signal, $n$ and $m$ are the number of past outputs and inputs of the plant depending on the plant order. In the case, when the input in (12) is used to control nonlinear discrete time system in (13), the problem of the output error will arise. To keep the plant output $y(k)$ convergence to the reference model output $y_{m}(k)$, we synthesize the control input $u(k)$ by the following equation:

$$
u(k)=v(k)+\bar{v}(k)
$$

where, $v(k)$ is the output of the adaptive controller and $\bar{v}(k)$ is the output of the NN. Then we derive $v(k)$ and $\bar{v}(k)$ as follows:

$$
\begin{aligned}
v(k)= & \frac{D(z) y_{m}(k+d)-\bar{R}(z) y(k)}{\bar{B}(z) \bar{S}(z)} \\
\bar{v}(k)= & f N\left(v(k-d), y_{m}(k+d), y(k-1), \ldots, y(k-n),\right. \\
& u(k-d), \ldots, u(k-d-m))
\end{aligned}
$$

where $f N($.) is the $\mathrm{NN}$ function.

The block diagram of the single input single output (SISO) nonlinear MRAC with NN is shown in Fig. 2. The control input is given by the sum of the output of the adaptive controller and the output of the NN. The inputs of the $\mathrm{NN}$ are given by the output of plant $y(k)$, the output of the reference model $y_{m}(k)$, the output of the adaptive control $v(k)$ and the input of the plant $u(k)$. The method of training is done by adjusting the weights of the NN until the output error $\lim _{k \rightarrow \infty}|e(k)|=\lim _{k \rightarrow \infty}\left|y_{m}(k)-y(k)\right| \leq \varepsilon$ is met.

\section{1 ${ }^{k \rightarrow \infty}$ Structure of the NN}

The $\mathrm{NN}$ is used to compensate for the nonlinearity of the plant dynamics that is not taken into consideration in

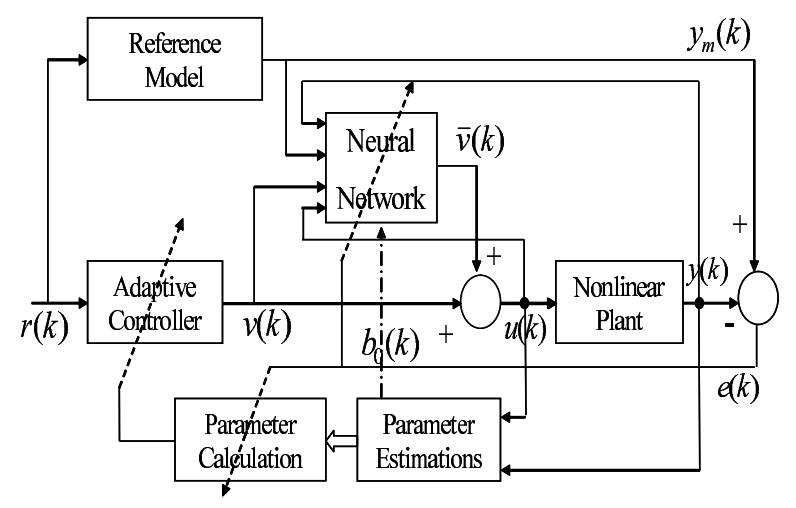

Fig. 2 Structure of the use of NN in MRAC 


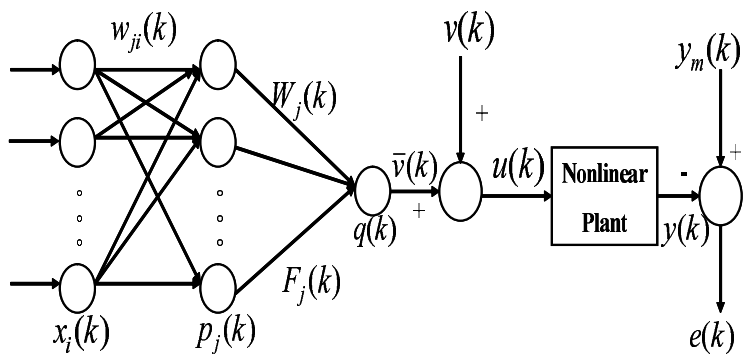

Fig. 3 Structure of the NN

the conventional MRAC. The role of the NN is to construct a linearized model by minimizing the output error caused by the nonlinearities in the control system. The multilayer backpropagation network has a very complex connection topology where every input is connected to all nodes in the first hidden layer, and every node in the hidden layers is connected to all nodes in the next layer, and so on. The multilayer backpropagation networks is especially useful for this purpose, because of its inherent nonlinear mapping capabilities ${ }^{(8)}$. Furthermore, the number of neurons in the hidden layer determines the complexity of the network. More hidden layers mean more connection between neurons and more weights that have to be trimmed. As a consequence, we need larger training sets. There were several propositions on how to calculate the necessary number of hidden neurons for a given problem. But these attempts yield contradictory results and have no practical importance. In practice, one starts with a given number of neurons in the hidden layers, and this number is varied and one tries to optimize the behavior of the network. The goal is to obtain a network with a high ability to generalize. This means that the error of the plant output has to be minimized.

Figure 3 shows our proposed NN which has 3 layers: an input layer, a hidden layer and an output layer with one neuron. Let $x_{i}(k)$ be the input to the $i$-th node in the input layer, $p_{j}(k)$ be the input to the $j$-th node in the hidden layer, $F_{j}$ be the output of the hidden layer. $q(k)$ be the input to the node in the output layer. Furthermore, let $w_{j i}$ be the weights between the input layer and the hidden layer, $W_{j}$ be the weight between the hidden layer and the output layer. Then the relations between the input layer and the output layer can expressed as follow

$$
\begin{aligned}
& p_{j}(k)=\sum_{i} w_{j i}(k) x_{i}(k) \\
& F_{j}(k)=f\left(p_{j}(k)\right) \\
& q(k)=\sum_{j} W_{j}(k) F_{j} \\
& \bar{v}(k)=f(q(k))
\end{aligned}
$$

where $f($.$) is the activation function. We choose the sig-$ moid function for the activation function as follows:

$$
f(x)=\frac{2 a}{1+\exp (-\mu x)}-a
$$

where $\mu>0$ and $a$ is a specified constant such that $a>0$, and $f(x)$ satisfies $-a<f(x)<a$. The derivative of Eq. (21) can be written as

$$
f^{\prime}(x)=\frac{\mu}{2 a}(a-f(x))(a+f(x))
$$

The input $x_{i}(k)$ to the $\mathrm{NN}$ is given by

$$
x_{i}(k) \equiv\left\{v(k), y_{m}(k), y(k), u(k)\right\}
$$

\subsection{Learning of the $\mathrm{NN}$}

The aim of training is to minimize the sum of the square error energy function

$$
E(k)=\frac{1}{2}\left[y_{m}(k)-y(k)\right]^{2}
$$

The weights in the output and the hidden layers are updated by using

$$
\begin{aligned}
W_{j}(k) & =W_{j}(k-1)+\Delta W_{j}(k) \\
& =W_{j}(k-1)+\eta\left(-\frac{\partial E(k)}{\partial W_{j}(k)}\right) \\
w_{j i}(k) & =w_{j i}(k-1)+\Delta w_{j i}(k) \\
& =w_{j i}(k-1)+\eta\left(-\frac{\partial E(k)}{\partial w_{j i}(k)}\right)
\end{aligned}
$$

where $\eta$ is the learning rate, $\frac{\partial E(k)}{\partial W_{j}(k)}$ and $\frac{\partial E(k)}{\partial w_{j i}(k)}$ can be obtained as follows:

$$
\begin{aligned}
\frac{\partial E(k)}{\partial W_{j}(k)}= & \frac{\partial E(k)}{\partial y(k)} \cdot \frac{\partial y(k)}{\partial u(k-d)} \cdot \frac{\partial u(k-d)}{\partial f(q(k))} \cdot \frac{\partial f(q(k))}{\partial q(k)} \\
& \cdot \frac{\partial q(k)}{\partial W_{j}(k)} \\
\frac{\partial E(k)}{\partial w_{j i}(k)}= & \frac{\partial E(k)}{\partial y(k)} \cdot \frac{\partial y(k)}{\partial u(k-d)} \cdot \frac{\partial u(k-d)}{\partial f(q(k))} \cdot \frac{\partial f(q(k))}{\partial q(k)} \\
& \cdot \frac{\partial q(k)}{\partial f\left(p_{j}(k)\right)} \cdot \frac{\partial f\left(p_{j}(k)\right)}{\partial p_{j}(k)} \cdot \frac{\partial p_{j}(k)}{\partial w_{j i}(k)}
\end{aligned}
$$

where

$$
\begin{aligned}
& \frac{\partial E(k)}{\partial y(k)}=-\left(y_{m}(k)-y(k)\right), \\
& \frac{\partial u(k-d)}{\partial f(q(k))}=1, \quad \frac{\partial q(k)}{\partial W_{j}(k)}=f\left(p_{j}(k)\right), \\
& \frac{\partial q(k)}{\partial f\left(p_{j}(k)\right)}=W_{j}(k), \quad \frac{\partial p_{j}(k)}{\partial w_{j i}(k)}=x_{i}(k), \\
& \frac{\partial f(q(k))}{\partial q(k)}=\frac{\mu}{2 a}(a-f(q(k)))(a+f(q(k))), \\
& \frac{\partial f\left(p_{j}(k)\right)}{\partial p_{j}(k)}=\frac{\mu}{2 a}\left(a-f\left(p_{j}(k)\right)\right)\left(a+f\left(p_{j}(k)\right)\right)
\end{aligned}
$$

also, $\partial y(k) / \partial u(k-d)$ is given by

$$
\begin{aligned}
\frac{\partial y(k)}{\partial u(k-d)} & =\frac{\partial y(k)}{\partial v(k-d)} \cdot \frac{\partial v(k-d)}{\partial u(k-d)} \\
& =\frac{\partial y(k)}{\partial v(k-d)} / \frac{\partial u(k-d)}{\partial v(k-d)}
\end{aligned}
$$

Let, $u(k-d)=v(k-d)+\bar{v}(k-d)$ then,

$$
\frac{\partial u(k-d)}{\partial v(k-d)}=1+\frac{\partial \bar{v}(k-d)}{\partial v(k-d)}
$$


According to (17), (18), (19), (20), and (23), $\partial \bar{v}(k-$ d) $/ \partial v(k-d)$ can be obtained as

$$
\begin{gathered}
\frac{\partial \bar{v}(k-d)}{\partial v(k-d)}=\frac{\partial f(q(k))}{\partial x_{1}(k)}=\frac{\partial f(q(k))}{\partial q(k)} \cdot \frac{\partial q(k)}{\partial f\left(p_{j}(k)\right)} \\
\cdot \frac{\partial f\left(p_{j}(k)\right)}{\partial p_{j}(k)} \cdot \frac{\partial p_{j}(k)}{\partial x_{1}(k)}
\end{gathered}
$$

Here $\partial p_{j}(k) / \partial x_{1}(k)=w_{1 j}(k)$

Furthermore, the linear model of the plant is constructed using estimated parameters. The estimated output of this linear model is $\bar{y}(k)$. We assume $y(k) \cong \bar{y}(k)$, and the approximate value of $\partial \bar{y}(k) / \partial v(k-d)$ can be calculated as below. Using a linear model as in (1), we can write

$$
\begin{aligned}
\bar{y}(k)= & -\sum_{i=1}^{n} \bar{a}_{i} \bar{y}(k-i)+\sum_{j=0}^{m} \bar{b}_{j} v(k-d-j) \\
= & -\bar{a}_{1} \bar{y}(k-1)-\bar{a}_{2} \bar{y}(k-2)+\ldots-\bar{a}_{n} \bar{y}(k-n) \\
& +\bar{b}_{0} v(k-d)+\bar{b}_{1} v(k-d-1)+\ldots \\
& +\ldots+\bar{b}_{m} v(k-d-m)
\end{aligned}
$$

From (32), we obtain

$$
\frac{\partial y(k)}{\partial v(k-d)} \cong \frac{\partial \bar{y}(k)}{\partial v(k-d)}=\bar{b}_{0}(k)
$$

then from (30), (31), and (33) it is possible to rewrite (29) as

$$
\frac{\partial y(k)}{\partial u(k-d)}=\frac{\bar{b}_{0}(k)}{1+H(k)}
$$

where $H(k)=\frac{\partial f\left(q_{1}(k)\right)}{\partial q_{1}(k)} \cdot \frac{\partial q_{1}(k)}{\partial f\left(p_{j}(k)\right)} \cdot \frac{\partial f\left(p_{j}(k)\right)}{\partial p_{j}(k)} \cdot \frac{\partial p_{j}(k)}{\partial x_{1}(k)}$.

From (34), we can verify that our proposed structure will give an efficient method for calculating the derivative of the system output with respect to the input using one identified parameter $\bar{b}_{0}$ in the MRAC and the internal variables of the $\mathrm{NN}(H(k))$, which enables to perform the backpropagation algorithm very efficiently.

\section{Stability Analysis}

From Fig. 2 we can verify that our proposed method utilize 2 types of controllers. The conventional controller based on linear model and the neural network controller. Both controllers build a synergy controllers that make a powerful adaptive control system. On the other hand, when discussing adaptive control systems, it is important to prove the stability of the system. Therefore, we would like to discuss the theoretical considerations about the global stability of the system.

\subsection{Stability of the conventional MRAC}

Global convergence for adaptive control algorithms (4), and (5) have been proven in reference ${ }^{(15),(16)}$. It is shown that algorithms will ensure that the system inputs and outputs remain bounded for all time and that the output tracking error converges to zero. The discrete time conventional MRAC described in section 2 are stable, according to references ${ }^{(15),(16)}$ if

1. The process time delay $d$ is known.
2. The reference model time delay is equal to or greater than the process time delay.

3. An upper bound on process order $n$ and $m$ are known.

4. The process is of minimum phase.

5. The estimated parameter $\bar{b}_{0} \neq 0$.

\subsection{Stability of the NN}

The basic idea of our analysis is to introduce a global stability criterion into the design procedure and to choose the adaptive law in such a away that the requirements of the stability criterion are fulfilled. We define |.| as euclidean norm for vector, and $\|$.$\| as frobenius norm for ma-$ trix ${ }^{(17)}$. The error between the reference model output and the plant output is given by

$$
e(k)=y_{m}(k)-y(k)
$$

and the sequential error can be expressed as

$$
\begin{aligned}
e(k+1) & =e(k)+\Delta e(k) \\
& =e(k)+\left[\frac{\partial e(k)}{\partial W(k)}\right]^{T} \Delta W(k)
\end{aligned}
$$

$\Delta W(k)$ can be expressed as

$$
\begin{aligned}
\Delta W(k) & =-\eta \frac{\partial E(k)}{\partial W(k)}=-\eta e(k) \frac{\partial e(k)}{\partial W(k)} \\
& =\eta e(k) \frac{\partial y(k)}{\partial W(k)}=\eta e(k) \frac{\partial y(k)}{\partial u(k-d)} \frac{\partial u(k-d)}{\partial W(k)} \\
& =\eta e(k) y_{s}(k) \frac{\partial \bar{v}(k-d)}{\partial W(k)}
\end{aligned}
$$

where $\frac{\partial y(k)}{\partial u(k-d)}=y_{s}(k)$ is the sensitivity of the plant, $W(k)$ and $\eta$ represent the weight and the learning rate, respectively. $\bar{v}(k)$ is the output of the $\mathrm{NN}$.

Since $\frac{\partial e(k)}{\partial W(k)}=-y_{s}(k) \frac{\partial \bar{v}(k-d)}{\partial W(k)}$ and from (37) $\Delta e(k)$ can be obtained:

$$
\Delta e(k)=-\eta y_{s}^{2}(k) e(k)\left\|\frac{\partial \bar{v}(k-d)}{\partial W(k)}\right\|^{2}
$$

Let us consider a discrete form of Lyapunov function as follows:

$$
V(k)=\frac{1}{2} e^{2}(k)
$$

From (36) and (38) $\Delta V$ will be determined as follows,

$$
\begin{aligned}
\Delta V= & V(k+1)-V(k) \\
= & \frac{1}{2}\left[e^{2}(k+1)-e^{2}(k)\right] \\
= & e(k) \Delta e(k)+\frac{1}{2} \Delta e^{2}(k) \\
= & -e(k) \eta y_{s}^{2}(k) e(k)\left\|\frac{\partial \bar{v}(k-d)}{\partial W(k)}\right\|^{2} \\
& +\frac{1}{2}\left\{-\eta y_{s}^{2}(k) e(k)\left\|\frac{\partial \bar{v}(k-d)}{\partial W(k)}\right\|^{2}\right\}^{2} \\
= & -e^{2}(k)\left\{\eta y_{s}^{2}(k)\left\|\frac{\partial \bar{v}(k-d)}{\partial W(k)}\right\|^{2}\right.
\end{aligned}
$$




$$
\begin{aligned}
& \left.-\frac{1}{2} \eta y_{s}^{4}(k)\left\|\frac{\partial \bar{v}(k-d)}{\partial W(k)}\right\|^{4}\right\} \\
\equiv & -\gamma e^{2}(k) .
\end{aligned}
$$

From (40) we know that $V$ is a nonincreasing sequence and also $V$ is bounded, and $\lim _{k \rightarrow \infty} V(k)$ exists. Therefore $\lim _{k \rightarrow \infty}|e(k)|=0$. Again, to make $\Delta V$ negative, $\gamma$ has to be positive $(\gamma>0)$. Let $\beta(k)=\frac{\partial \bar{v}(k-d)}{\partial W(k)}, y_{s, \text { max }}:=$ $\max _{k}\left|y_{s}(k)\right|, \beta_{\max }=\max _{k} \mid \beta(k) \|$, and $\eta=\frac{\eta_{1}}{\beta_{\max }^{2} y_{s, \max }^{2}}$ then

$$
\begin{aligned}
\gamma & =\frac{1}{2} y_{s}^{2}(k)\|\beta(k)\|^{2} \eta\left(2-\eta y_{s}^{2}(k)\|\beta(k)\|^{2}\right) \\
& =\frac{1}{2} y_{s}^{2}(k)\|\beta(k)\|^{2} \eta\left(2-\eta_{1} \frac{y_{s}^{2}(k)\|\beta(k)\|^{2}}{\beta_{\max }^{2} y_{s, \max }^{2}}\right) \\
& =\frac{1}{2} y_{s}^{2}(k)\|\beta(k)\|^{2} \eta\left(2-\eta_{1}\right)>0
\end{aligned}
$$

From (41), we obtain $0<\eta_{1}<2$, then the convergence of $\eta$ is guaranteed if

$$
0<\eta<\frac{2}{y_{s, \max }^{2} \beta_{\max }^{2}}
$$

From (19), and (20), the gradient with respect to the output weight $W_{j}$ can be determined

$$
\beta^{W}(k)=\frac{\partial \bar{v}(k-d)}{\partial W_{j}(k)}=\frac{\partial \bar{v}(k-d)}{\partial q(k)} \frac{\partial q(k)}{\partial W_{j}(k)}=\dot{f}(q(k)) F_{j}(k)
$$

where $\dot{f}$ is the derivative of the sigmoid function in (21), $F_{j}$ is the output value of the $j$ th neuron in the hidden layer. In this research, we selected $a=6.7$ and $\mu=0.1$ for the sigmoid function in (21), therefore $-6.7<F_{j}<6.7$ $\left(j=1,2, \ldots, n_{j}\right)$, and $0<\dot{f}<0.335, F_{j}=\left[F_{1}, \ldots, F_{n_{j}}\right]^{T}, n_{j}$ is the number of neurons in the hidden layer. Then $F_{j, \max }:=\max _{k}\left\|F_{j}(k)\right\|,\left\|F_{j}(k)\right\|:=\max _{j}\left|F_{j}(k)\right|=6.7 \sqrt{n_{j}}$, $\dot{f}(q)_{\text {, } \max }:=\max _{k}|\dot{f}(q(k))|=0.335$, Furthermore we can calculate $\beta_{\max }^{W}:=\max _{k}\left\|\beta^{W}(k)\right\|$

$$
\beta_{\max }^{W}=\dot{f}(q)_{\text {, } \max } F_{j, \text { max }}=2.2445 \sqrt{n_{j}}
$$

From (34) we obtain

$$
y_{s}(k)=\frac{\partial y(k)}{\partial u(k-d)}=\frac{\bar{b}_{0}(k)}{1+H(k)}
$$

where $H(k)$ be positive constant and if $H(k) \simeq 0$ then $y_{s}(k) \simeq \bar{b}_{0}(k)$. Let $y_{s, \max }:=\max _{k}\left|y_{s}(k)\right|$,

$$
y_{s, \max }(k) \simeq \bar{b}_{0}(k)
$$

from (42), (44), and (45), we can determine

$$
0<\eta^{W}<\frac{0.4}{\bar{b}_{0}^{2} n_{j}}
$$

From (17), (18), (19), and (20), the gradient with respect to the input weight $w_{j i}$ can be determined

$$
\begin{aligned}
\beta^{w}(k)=\frac{\partial \bar{v}(k-d)}{\partial w_{j i}(k)} & =\frac{\partial \bar{v}(k-d)}{\partial q(k)} \frac{\partial q(k)}{\partial F_{j}(k)} \frac{\partial F_{j}(k)}{\partial p_{j}(k)} \frac{\partial p_{j}(k)}{\partial w_{j i}(k)} \\
& =\dot{f}(q(k)) W_{j}(k) \dot{f}\left(p_{j}(k)\right) x_{i}(k)
\end{aligned}
$$

where $W_{j}(k)$ and $x_{i}(k)$ are the weight value of the output layer and the input value, respectively.

Again, let $a=6.7$ and $\mu=0.1$ be used for the sigmoid function in (21), therefore $0<\dot{f}<0.335, \dot{f}\left(p_{j}\right)=$ $\left[\dot{f}\left(p_{1}\right), \ldots, \dot{f}\left(p_{n_{j}}\right)\right]^{T}, n_{j}$ is the number of neurons in the hidden layer. Let $\dot{f}\left(p_{j}\right)_{\text {,max }}:=\max _{k}\left\|\dot{f}\left(p_{j}(k)\right)\right\|,\left\|\dot{f}\left(p_{j}(k)\right)\right\|$ $:=\max _{j}\left|\dot{f}\left(p_{j}(k)\right)\right|$, then $\dot{f}\left(p_{j}\right)_{\text {, } \max }=0.335 \sqrt{n_{j}}$, and $\dot{f}(q)_{\text {, } \max }:=\max _{k}|\dot{f}(q(k))|=0.335$, also $W_{j, \text { max }}:=\operatorname{mak}_{k}$ $\left\|W_{j}(k)\right\|$ and $x_{i, \max }:=\max _{k}|x(k)|$.

Furthermore, let $\beta_{\max }^{w}:=\max _{k}\left\|\beta^{w}(k)\right\|$ we can determine

$$
\beta_{\max }^{w}=0.1122 \sqrt{n j} W_{j, \max } x_{i, \max }
$$

From (42), (46), and (49) we can determine

$$
0<\eta^{w}<\frac{159}{n_{j}\left[\bar{b}_{0} W_{j, \max } x_{i, \max }\right]^{2}}
$$

\section{Experimental Magnetic Levitation System}

The magnetic levitation test bed which is used for our experiment is shown in Fig. 4. This system has inherent strong nonlinearities due to the natural properties of magnetic fields and uncertainties. The magnetic levitation system consists of upper and lower drive coils that produce a magnetic field in response to a DC current. The levitated magnet travel along a precision glass guide rod as shown in Fig. 4. By energizing the lower coil, a single magnet is levitated through a repulsive magnetic force. Moreover, the magnets are of an ultra-high field strength rare earth $(\mathrm{NeBFe})$ type and are designed to provide large levitated displacements to clearly demonstrate the principle of levitation and motion control ${ }^{(19)}$.

The free body diagram of two suspended magnets in the Model 730 apparatus is shown in Fig. 5. For Model 730 apparatus, the friction forces $C_{1} \dot{y}_{1}$ is typically small. Therefore, from Fig. 5, the following equation of motion for the levitation system can be simply derived according to force balance analysis in the vertical plane as

$$
m \ddot{y}=F_{u}-m g
$$

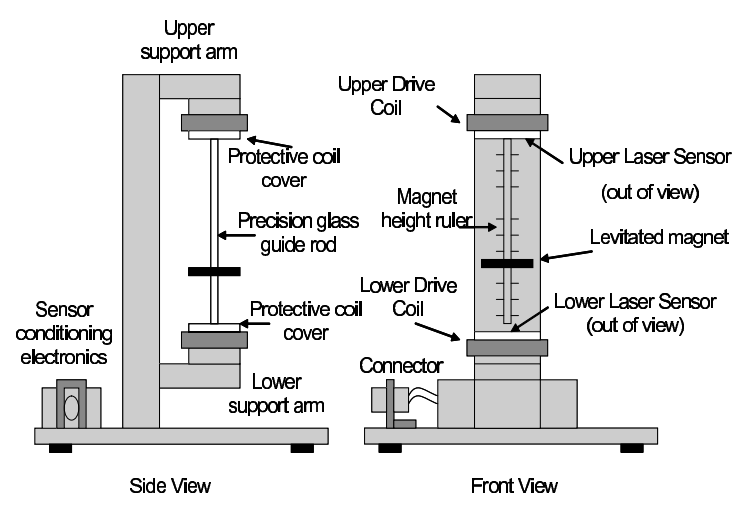

Fig. 4 The Model 730 magnetic levitation system test bed ${ }^{(19)}$ 


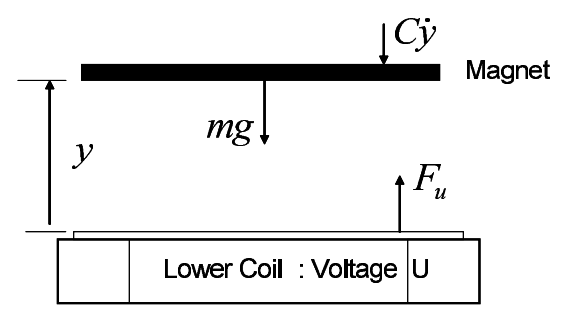

Fig. 5 Force diagram for the magnetic levitation system

where $m$ is the mass of the levitation magnet, $y$ is the distance of the levitation magnet, and $F_{u}$ is the magnetic force term that are modeled as having the following form

$$
F_{u}=\frac{i}{a_{1}\left(y+a_{2}\right)^{4}}
$$

where $a_{1}$ and $a_{2}$ are constants that can be determined by numerical modeling of the magnetic configuration, and $i$ is the current of the coil.

Here, we replace the coil current $i$ to the more general term, denoted as $u$. The general term may be a digital word, voltage, or current and is presumed to be linearly proportional to the coil current. The coefficient $a_{1}$ must of course be consistently scaled with the units of $u$. In this paper, we consider $u$ to be a voltage, therefore (52) are redefined as

$$
F_{u}=\frac{u}{a_{1}\left(y+a_{2}\right)^{4}}
$$

On the other hand, for small motions, the system may be modeled as being linear. Hence, the above system can be simply linearized at the equilibrium operating as following state-space representation [see Appendix 2].

$$
\begin{aligned}
& \dot{\boldsymbol{x}}(t)=A \boldsymbol{x}(t)+\boldsymbol{b} u(t) \\
& y(t)=\boldsymbol{c x}(\boldsymbol{t})
\end{aligned}
$$

where

$$
\boldsymbol{x}(t)=\left[\begin{array}{c}
y_{1}^{*} \\
\dot{y}_{1}^{*}
\end{array}\right], A=\left[\begin{array}{cc}
0 & 1 \\
-\frac{k_{1}^{\prime}}{m} & 0
\end{array}\right], \boldsymbol{b}=\left[\begin{array}{c}
0 \\
\frac{k_{2}^{\prime}}{m}
\end{array}\right], \boldsymbol{c}=\left[\begin{array}{l}
1 \\
0
\end{array}\right]
$$

In this paper we will focus in discrete systems which can be represented by the difference equation corresponding to the differential equation given in (54). If the system described by (54) is assumed linear and time invariant, the equations in (54) can be expressed as

$$
\begin{aligned}
& \boldsymbol{x}(k+1)=\boldsymbol{A} \boldsymbol{x}(k)+\boldsymbol{b} u(k) \\
& y(k)=\boldsymbol{c} \boldsymbol{x}(k)
\end{aligned}
$$

For SISO plant, the matrix $\boldsymbol{A}$ and vector $\boldsymbol{b}$ and $\boldsymbol{c}$ in (55) can be chosen in such a fashion that the plant equation can be written as $(1)^{(7)}$.

The experimental setup for this experiment is shown in Fig. 6. The computer system is used to run the education control product (ECP) execute program and communicates with the ECP's digital signal processing (DSP) based real time controller. The real time controller unit contains the DSP, servo/actuator interfaces, servo amplifier

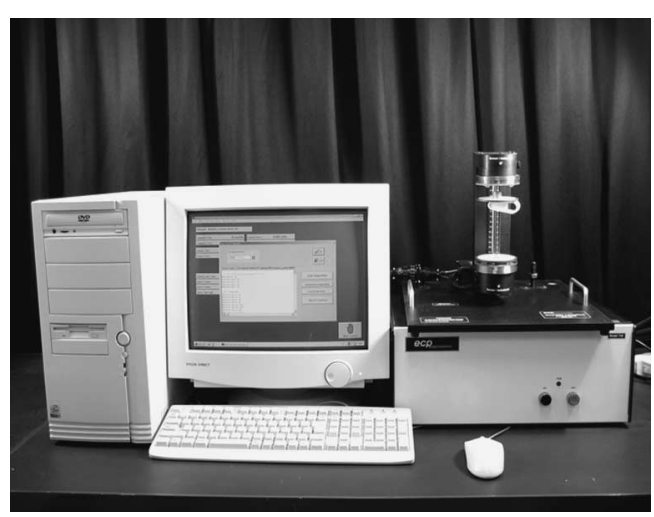

Fig. 6 Experimental setup

and auxiliary power supplies. Four 16 bit analog to digital converters (ADCs) are used to digitize the laser sensor signals. Two optional auxiliary digital to analog converters (DACs) provide for real time analog signal measurement.

\section{Experimental Results}

The magnetic levitation system which is introduced in the previous section is utilised to confirm the effectiveness of the proposed MRAC control strategy. The system is tested using the proposed control strategy and compared with the conventional MRAC. Both controllers are implemented at a sampling time of $4.420 \mathrm{msec}$.

Figure 7 and 8 show the results of using conventional MRAC, where the dashed line shows reference model output and the solid line shows the plant output. The horizontal axis shows the time in seconds and the vertical axis shows the height of the levitated magnet in centimeters. In Fig. 7 , we utilise 3 parameter estimations $\left(a_{1}, a_{2}, b_{0}\right)$, the result shows that the output of the plant can not follow the reference model output and after $1 \mathrm{~s}$ oscillation occurs. The system can not recognize the plant with only 3 parameter estimations. In Fig. 8 we utilise 5 parameter estimations $\left(a_{1}, a_{2}, a_{3}, b_{0}, b_{1}\right)$, the result shows that the plant output tries to follow the reference model output but errors still occur. High error occurs especially when the reference model output is steady at $2 \mathrm{~cm}$. The output of the plant is also steady at $1.7 \mathrm{~cm}$, consequently it can not converge to the reference model output. From (1) we can observe that more parameter estimations is better for estimating the system. Moreover, to estimate parameters of a nonlinear system with a linear model requires a lot of parameter estimations. Using a lot of parameter estimations means higher complexity, more calculations, and time consuming. On the other hand, we should consider the specifications of the system. Unfortunately, using more than 5 parameter estimations in the magnetic levitation system is impossible due to the limitation of the system specification.

Figures 9-11 show the results when we are using our 


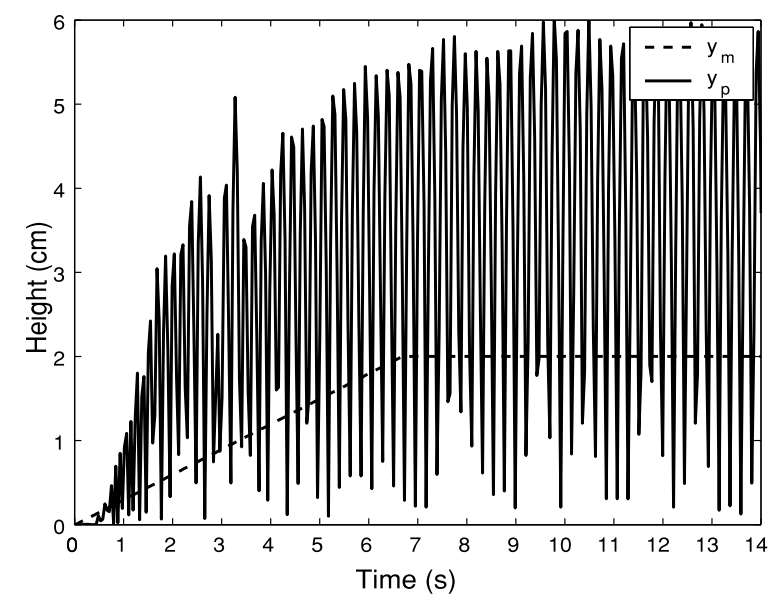

Fig. 7 The reference model output $y_{m}(k)$ and the plant output $y(k)$ when using conventional MRAC with 3 parameter estimations

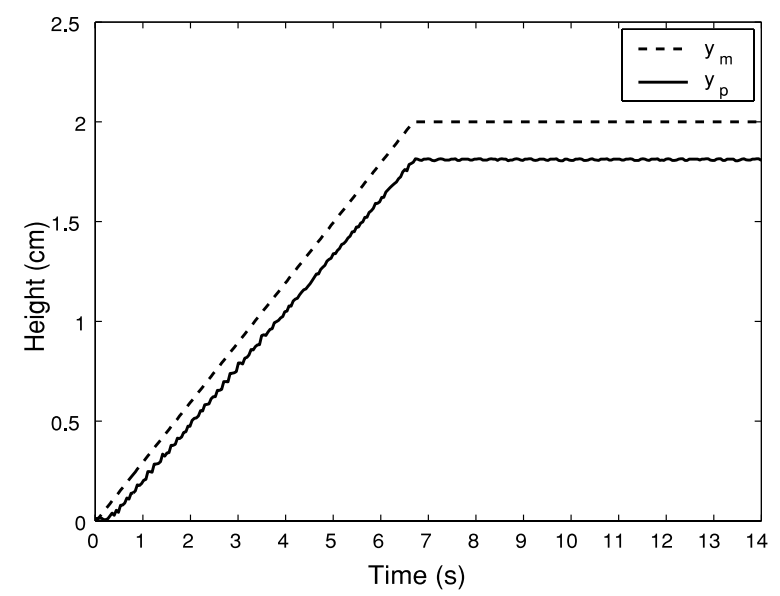

Fig. 8 The reference model output $y_{m}(k)$ and the plant output $y(k)$ when using conventional MRAC with 5 parameter estimations

proposed method where the learning rate is set to $1,0.5$ and 100 respectively. The neural network employs 4 neurons in both of the hidden and the output layers. When the learning rate is set to 1 , we get the system response as shown in Fig. 9, where at the period from $0 \mathrm{~s}$ to $4 \mathrm{~s}$, the result looks similar to result of using the conventional method (Fig. 8), then the output of the plant tries to follow the reference model output (at period from $4 \mathrm{~s}$ to $7 \mathrm{~s}$ ). Although the reference model output is steady, the output of the plant attempts to meet the reference model output (at period from $7 \mathrm{~s}$ to $11 \mathrm{~s}$ ). The output of the plant can converge to the reference model output smoothly after 11 seconds. This is an advantage in our control strategy that can not be achieved in the conventional method. When the learning rate is set to 0.5 , the output of the plant tracks the reference model output more slowly as shown in Fig. 10. Favourably, the system continues tracking the reference model output even at the steady condition. Comparing to the conventional method, our proposed method

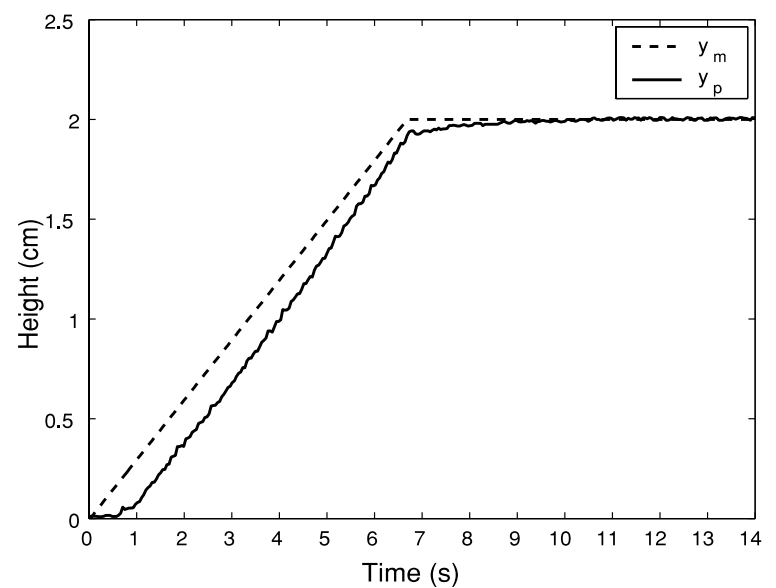

Fig. 9 The reference model output $y_{m}(k)$ and the plant output $y(k)$ when using NN in MRAC with $\eta=1$

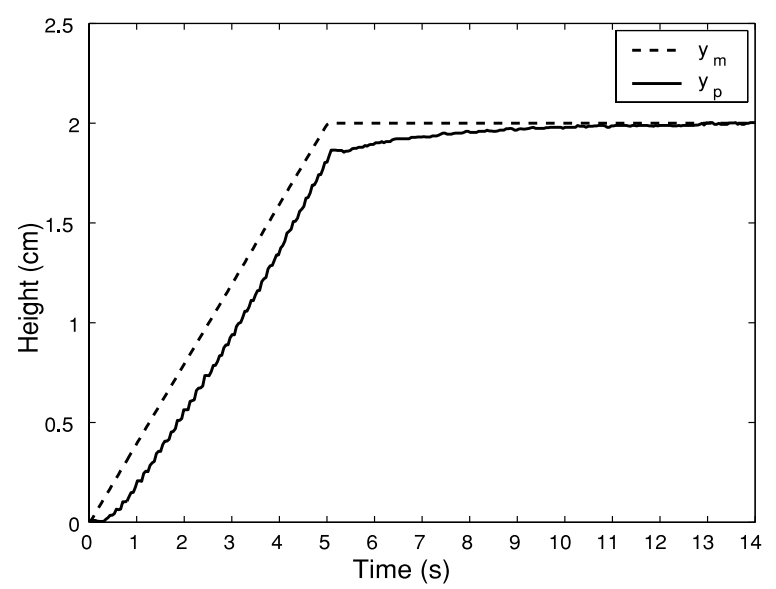

Fig. 10 The reference model output $y_{m}(k)$ and the plant output $y(k)$ when using NN in MRAC with $\eta=0.5$

can learn to track the output of the reference model more closely. When the learning rate is set to 100 the output of plant tracks the reference model output faster than the other cases as shown in Fig. 11. Unfortunately, after 8 seconds the output becomes unstable. It is clear that the good choice of the learning rate is very important.

In Fig. 12, we employ 2 hidden layer neurons in our proposed method with $\eta=1$ is used. Comparing the results in Figs. 12 and 9 (using 4 hidden layer neurons), the controller performance is better especially at the period from $4 \mathrm{~s}$ to $9 \mathrm{~s}$, but after $10 \mathrm{~s}$ the plant output can not match the reference model output. The number of neurons in the hidden layer is responsible for the characteristics of the neural network. When, the number of hidden neurons is too small, the neural network is not able to learn. If the number of hidden neurons is too large, the ability to generalize is lost. More hidden neurons mean higher complexity and more parameters that have to be determined. As a consequence, it needs larger training sets. There are many different aspects that play a role for the design of the hidden layer. Specification of the system is one aspect of consid- 


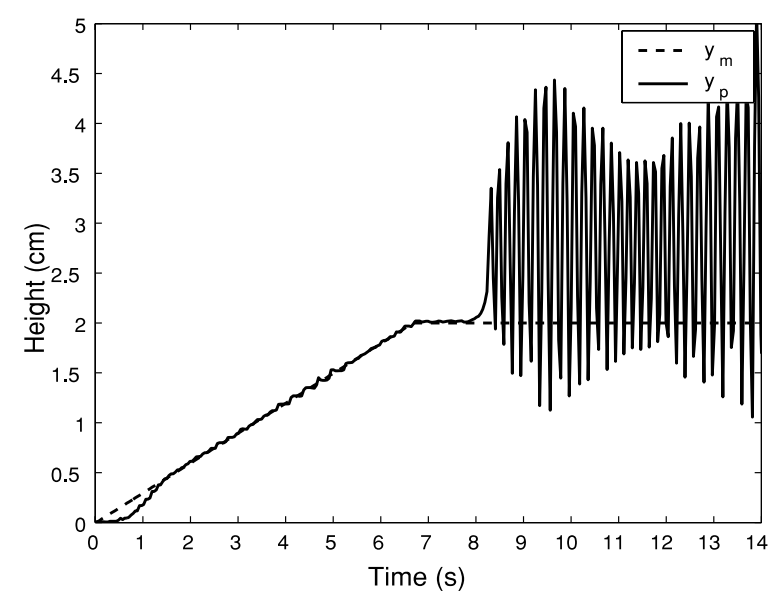

Fig. 11 The reference model output $y_{m}(k)$ and the plant output $y(k)$ when using NN in MRAC with $\eta=100$

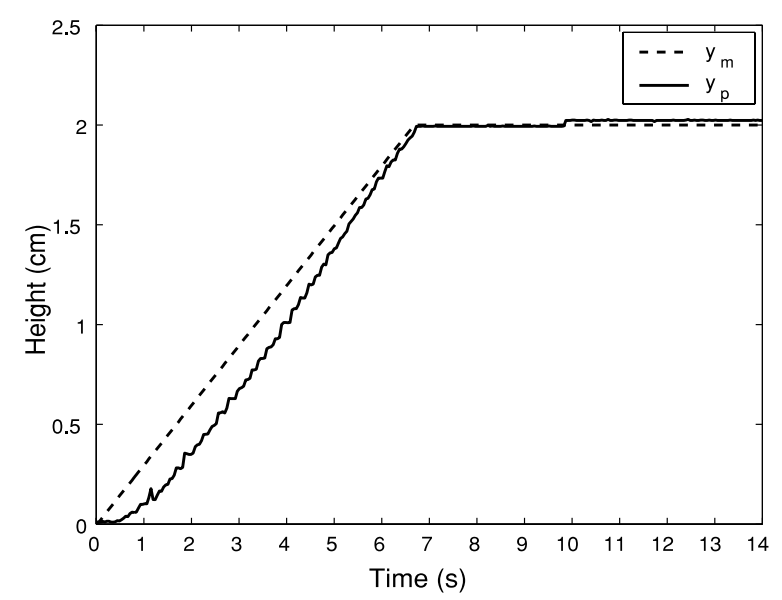

Fig. 12 The reference model output $y_{m}(k)$ and the plant output $y(k)$ when using $\mathrm{NN}$ in MRAC with 2 neurons in hidden layer

eration in the design of the hidden layer. Unfortunately, we can not implement a big number of hidden neurons in our proposed neural network because of the limitation of the system.

Figure 13 shows the results of using the conventional method and our proposed strategy. We inspect the strength of our proposed method $\left(y_{1}\right)$ compared to the conventional method $\left(y_{2}\right)$ with identical reference model output $\left(y_{m}\right)$ as shown in this figure. The conventional method uses 5 parameter estimations. The learning rate of the neural network is set to 1 . It can be seen that the conventional method can not control the whole period of the plant output. On the contrary, our proposed controller can control the whole period of the plant output. This means that our proposed method is more powerful than the conventional method.

Figure 14 shows the plant output with respect to the input plant. The horizontal axis show the time in seconds and the vertical axis shows the coil voltage in volt (for the input) and the height of the levitated magnet in centimeter

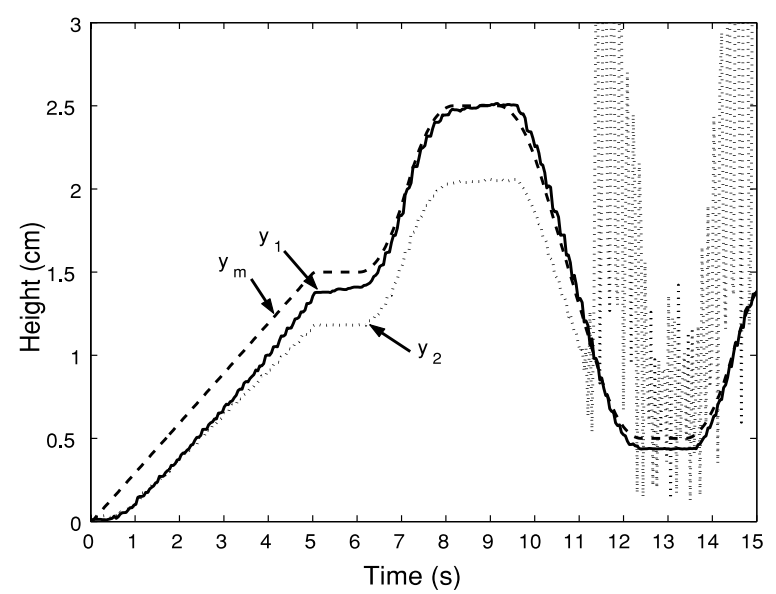

Fig. 13 The system output when using our proposed controller $y_{1}$ and conventional MRAC $y_{2}$

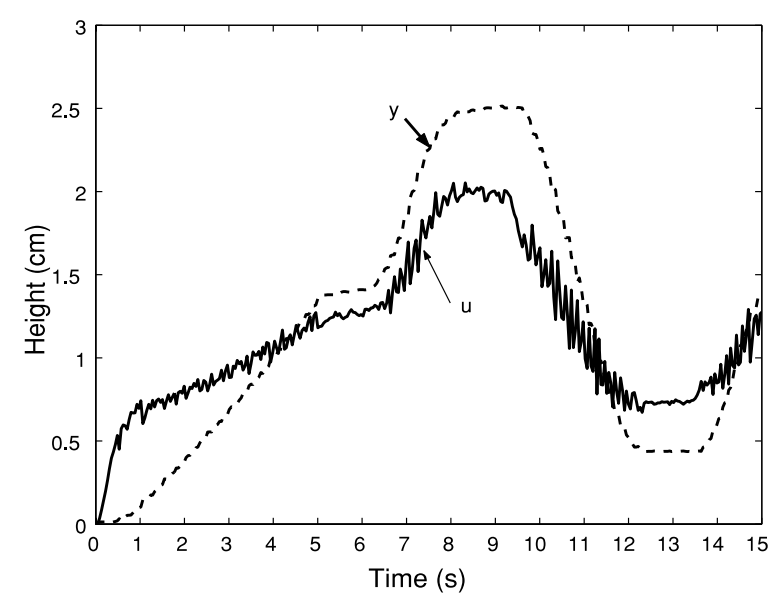

Fig. 14 The plant input $u(k)$ and the plant output $y(k)$

(for the output). From this figure we can see that control characteristic of the magnetic levitation system is nonlinear.

\section{Conclusions}

This research tried to point out the problems related to conventional MRAC for controlling the real plant of the nonlinear magnetic levitation system. Our proposed method utilized both adaptive controller and neural network controller systems that created a powerful adaptive control system. The input of the plant was given by the sum of the outputs of adaptive controller and the NN. The NN was used to compensate for the nonlinearity of the plant that is not taken into consideration in the conventional MRAC. We developed an efficient method for calculating the sensitivity of the plant using one identified parameter in the linearized model, and then its sensitivity was utilized in the $\mathrm{NN}$ to perform the backpropagation algorithm efficiently.

Furthermore, when discussing adaptive control, proof of asymptotical stability is necessary, therefore, we developed the theoretical consideration of the global stability 
of the system, and we proved that our proposed controller was guaranteed stable within our limitations.

Experimental comparison between a conventional controller and our proposed controller for controlling a magnetic levitation system was investigated. The experimental results showed that our proposed controller improved the tracking performance and reduced the error effectively.

\section{Appendix 1}

Let $\bar{D}(z)=1$, then there exist unique polynomials $\bar{R}(z)$ and $\bar{S}(z)$ which satisfy:

$$
\bar{D}(z)=\bar{A}(z) \bar{S}(z)+z^{-d} \bar{R}(z)
$$

where $\bar{A}(z)=1+\sum_{i=1}^{n} \bar{a}_{i} z^{-i}, \bar{S}(z)=1+\sum_{i=1}^{d-1} \bar{s}_{i} z^{-i}, \bar{R}(z)=\sum_{i=0}^{n-1} \bar{r}_{i} z^{-i}$ Then (56) can be solved as follows

$$
\begin{aligned}
& 1=\bar{A}(z) \bar{S}(z)+z^{-d} \bar{R}(z) \\
& 1=\left(1+\sum_{i=1}^{n} \bar{a}_{i} z^{-i}\right)\left(1+\sum_{i=1}^{d-1} \bar{s}_{i} z^{-i}\right)+z^{-d}\left(\sum_{i=0}^{n-1} \bar{r}_{i} z^{-i}\right) \\
& 1=\left(1+\bar{a}_{1} z^{-1}+\ldots+\bar{a}_{n} z^{-n}\right)\left(1+\bar{s}_{1} z^{-1}+\ldots\right. \\
& \left.+\bar{s}_{d-1} z^{-(d-1)}\right)+\bar{r}_{0} z^{-d}+\ldots+\bar{r}_{n-1} z^{-d-n+1} \\
& 1=1+\bar{a}_{1} z^{-1}+\ldots+\bar{a}_{n} z^{-n} \\
& +\bar{s}_{1} z^{-1}+\ldots+\bar{a}_{n-1} \bar{s}_{1} z^{-n}+\bar{a}_{n} \bar{s}_{1} z^{(-n+1)} \\
& + \text {. . } \\
& +. . \\
& + \text {. . } \\
& +\bar{s}_{d-1} z^{-(d-1)}+\ldots \\
& +\bar{a}_{n} \bar{s}_{d-1} z^{-(d+n-1)}+\bar{r}_{0} z^{-d}+\ldots \\
& +\bar{r}_{n-1} z^{-(d+n-1)}
\end{aligned}
$$

from (57) we can write

$$
\begin{aligned}
& 0=\bar{a}_{1}+\bar{s}_{1} \\
& 0=\bar{a}_{2}+\bar{a}_{1} \bar{s}_{1}+\bar{s}_{2} \\
& 0=\bar{a}_{3}+\bar{a}_{2} \bar{s}_{1}+\bar{a}_{1} \bar{s}_{2}+\bar{s}_{3} \\
& 0=\quad \cdot \quad \cdot \quad \cdot \\
& 0=\quad \cdot \quad \cdot \quad \cdot \\
& 0=\bar{a}_{n} \bar{s}_{d-3}+\bar{a}_{n-1} \bar{s}_{d-2}+\bar{a}_{n-2} \bar{s}_{d-1}+\bar{r}_{n-3} \\
& 0=\bar{a}_{n} \bar{s}_{d-2}+\bar{a}_{n-1} \bar{s}_{d-1}+\bar{r}_{n-2} \\
& 0=\bar{a}_{n} \bar{s}_{d-1}+\bar{r}_{n-1}
\end{aligned}
$$

where $\bar{a}_{n}(n=1, . ., n)$ are parameter estimations of the plant, then $\bar{s}_{i}(i=1, . . d-1)$ and $\bar{r}_{i}(i=0, . . n-1)$ can be obtained.

\section{Appendix 2}

The equation of motion for the magnetic levitation system is given as

$$
\begin{aligned}
m \ddot{y} & =F_{u}-m g \\
F_{u} & =\frac{u}{a_{1}\left(y+a_{2}\right)^{4}}
\end{aligned}
$$

When the nonlinear term (right hand side) of (59) is presented as $\alpha(y, u, t)$, we have

$$
m \ddot{y}=\alpha(y, u, t)=F_{u}-m g
$$

Then the linearized equation of motion is found by calculating

$$
\begin{aligned}
& \alpha(y, u, t)-\alpha\left(y_{0}, u_{0}, t\right)= \\
& \quad=\left.\frac{\partial \alpha}{\partial y}\right|_{y_{0}, u_{0}}\left(y-y_{0}\right)+\left.\frac{\partial \alpha}{\partial u}\right|_{y_{0}, u_{0}}\left(u-u_{0}\right)
\end{aligned}
$$

where $y_{0}, u_{0}$ are the magnet position and control effort that define the operating point. For the purposes of control design, we shall choose the operating point to be at an equilibrium so that

$$
\alpha\left(y_{0}, u_{0}, t\right)=F_{u}-\left.m g\right|_{y_{0}, u_{0}}=0
$$

Evaluating (62) and using (63) we have

$$
m \ddot{y}=-\frac{4 u_{0}}{a_{1}\left(y_{0}+a_{2}\right)^{5}}\left(y-y_{0}\right)+\frac{1}{a_{1}\left(y_{0}+a_{2}\right)^{4}}\left(u-u_{0}\right)
$$

which may be rewritten as

$$
m \ddot{y}^{*}+k_{1}^{\prime} y^{*}=k_{2}^{\prime} u^{*}
$$

where

$$
\begin{aligned}
& y^{*}=y-y_{0}, \quad u^{*}=u-u_{0} \\
& k_{1}^{\prime}=\frac{4 u_{0}}{a_{1}\left(y_{0}+a_{2}\right)^{5}}, \quad k_{2}^{\prime}=\frac{1}{a_{1}\left(y_{0}+a_{2}\right)^{4}}
\end{aligned}
$$

From (63) we may solve for the equilibrium control input values as

$$
u_{0}=a_{1} m g\left(y_{0}+a_{2}\right)^{4}
$$

Let $y^{*}=y_{1}^{*}$ and $\dot{y}^{*}=y_{2}^{*}$, then we get

$$
\left[\begin{array}{c}
\dot{y}_{1}^{*} \\
\ddot{y}_{2}^{*}
\end{array}\right]=\left[\begin{array}{cc}
0 & 1 \\
-\frac{k_{1}^{\prime}}{m} & 0
\end{array}\right]\left[\begin{array}{l}
y_{1}^{*} \\
y_{2}^{*}
\end{array}\right]+\left[\begin{array}{c}
0 \\
\frac{k_{2}^{\prime}}{m}
\end{array}\right] u^{*}
$$

\section{References}

( 1 ) Åström, K.J. and Wittenmark, B., Adaptive Control (2nd ed.), (1995), Addison-Wesley.

( 2 ) Parks, P.C., Liapunov Redesign of Model Reference Adaptive Control Systems, IEEE Trans on Automatic Control, Vol.AC-11, No.3 (1966), pp.362-367.

( 3 ) Hang, C.C. and Parks, P.C., Comparative Studies of Model Reference Adaptive Control System, IEEE Trans. Automatic Control, Vol.AC-18, No.5 (1973), pp.419-428.

(4) Landou, I.D., A Survey of Model Reference Adaptive Techniques Theory and Applications, Automatica, Vol.10 (1974), pp.353-379.

( 5 ) Lu, J., Phuah, J. and Yahagi, T., A Method of Model Reference Adaptive Control for MIMO Nonlinear System Using Neural Networks, IEICE Trans, Fundamentals, Vol.E84-A, No.8 (2001), pp.1933-1941.

( 6 ) Antsaklis, P.J., Neural Networks for Control Systems, IEEE Trans. Neural Networks, Vol.1, No.2 (1990), pp.242-244. 
( 7 ) Narendra, K.S. and Parthasarathy, K., Identification and Control of Dynamical System Using Neural Networks, IEEE Trans. Neural Networks, Vol.1, No.1 (1990), pp.4-27.

( 8 ) Warwick, K., Irwin, G.W. and Hunt, K.J., Neural Networks for Control and Systems, IEE Control Engineering Series 46, (1992).

(9) Noriega, J.R. and Wang, H., A Direct Adaptive Neural Network Control for Unknown Nonlinear Systems and Its Application, IEEE Trans. Neural Networks, Vol.9, No.1 (1998), pp.27-34.

(10) Gong J.Q. and Yao, B., Neural Network Adaptive Robust Control with Application to Precision Motion Control of Linear Motors,Int.J.Adapt.Control and Signal Processing, No.15 (2001), pp.837-864.

(11) Ahmed, M.S., Neural Net Based MRAC for a Class of Nonlinear Plants, Neural Networks, 13 (2000), pp.111124.

(12) Lightbody, G. and Irwin, G.W., Direct Neural Model Reference Adaptive Control, IEE Proc.Control Theory Appl, Vol.142, No.1 (1995), pp.31-43.

(13) Hagan, M.T., Demuth, H.B. and Jesus, O.D., An Intro- duction to the Use of Neural Networks in Control Systems, Int. J. Robust Nonlinear Control, Vol.12 (2002), pp.959-985.

(14) Cho, G., Kato, Y. and Spilman, D., Sliding Mode and Classical Controllers in Magnetic Levitation Systems, IEEE Control System Magazine, Vol.13 (1993), pp.4248.

(15) Goodwin, G.C., Ramadge, P.J. and Caines, P.E., Discrete Time Multivariable Adaptive Control, IEEE Trans. Automatic Control, Vol.AC-25, No.3 (1980), pp.449-456.

(16) Isermann, R., Lachmann, K.H. and Matko, D., Adaptive Control Systems, (1992), Prentice Hall (UK).

(17) Polycarpou, M.M. and Ioannou, P.A., Learning and Convengence Analysis of Neural-Type Structured Netwoks, IEEE Trans. Neural Networks, Vol.3, No.1 (1992), pp.39-50.

(18) Ku, C. and Lee, K.Y., Diagonal Recurrent Neural Networks for Dynamic Systems Control, IEEE Trans. Neural Networks, Vol.6, No.1 (1995), pp.144-155.

(19) Parks, T.R., Manual for Model 730 Magnetic Levitation System, (1991), Educational Control Products. 